\title{
USING CONTEXTUAL GRAPHS AS A DECISION-MAKING TOOL IN THE PROCESS OF HiRING CANDIDATES
}

\author{
Hassane Tahir ${ }^{1}$ and Patrick Brézillon ${ }^{2}$ \\ ${ }^{1}$ Links Consultants 24 Rue de Prony, 75017 Paris, France \\ ${ }^{2}$ Laboratory of Computer Science-Paris 6, Sorbonne University, Paris, France
}

\begin{abstract}
Poor selection of employees can be a first step towards a lack of motivation, poor performance, and high turnover, to name a few. It's no wonder that organizations are trying to find the best ways to avoid these slippages by finding the best possible person for the job. Therefore, it is very important to understand the context of hiring process to help to understand which recruiting mistakes are most damaging to the organization in order to reduce the recruiting challenges faced by Human resource managers by building their capacity to ensure optimal HR performance. This paper initiates a research about how Contextual Graphs Formalism can be used for improving the decision making in the process of hiring potential candidates. An example of a typical procedure for visualization of recruiting phases is presented to show how to add contextual elements and practices in order to communicate the recruitment policy in a concrete and memorable way to both hiring teams and candidates.
\end{abstract}

\section{KEYWORDS}

Contextual Graphs, Contextual Elements, Decision Making, Hiring Process, Practices, Procedure, Recruitment Policy.

\section{INTRODUCTION}

Poor employee selection can be a first step towards a lack of motivation, poor performance, and high turnover, to name a few. It is not surprising that organizations are trying to find the best ways to avoid these slippages by finding the best possible person for the job. Therefore, it is necessary to understand the context in which recruitment errors are more harmful to an organization; hiring a candidate whose application should have been rejected, or rejecting that of the candidate who should have been hired. The first could be a choice to fill a position quickly while the second could be because we have not seen the true potential of the person. This will assist resource managers to significantly reduce the recruitment problems they face and build their capabilities to ensure optimal HR performance. Other research on Big data helps efficiently to process data by using data mining algorithms in order improve decision making for human resources optimization and customer service improvement [18] [19].

Many companies invest a lot of resources and time interviewing external candidates because some of contextual elements are not considered [2]. Some of these contextual elements can be stated below:

- Do the company have the needed talents already present in-house? 
International Journal of Artificial Intelligence and Applications (IJAIA), Vol.11, No.5/6, November 2020

- What are the techniques (personality and psychometric tests, etc.) used in interview process to detect and build on these natural assets, something that may be less obvious to detect?

- Are social media platforms used? If yes, do the company have the resources available to screen applications and respond to the potentially high volume of applications! This can alleviate some recruitment problems.

- Do recruiter colleagues have different viewpoints about the candidate or share the same context?

- Do the company have the contextual elements for selecting best candidates (salary, experience, personality, university reputation, etc ...)? This will help a company in his decision making as for example choose between a freshly out-of-university candidate, whose personality profile suggests that he or she is motivated, interested and built for a sales position, or a candidate who has more experience in sales but whose innate personality does not match the competitive and results-oriented aspect that such a job requires.

- Can the candidate hold the open position under an exceptional context? (i.e. an epidemy like COVID 19)? In this case some candidates need to be properly evaluated to see if they are autonomous and can work in telework with other members of a team without a major constraint.

The above contextual elements are relevant at a given time and the values taken by these elements at the moment of hiring candidates by a company. A potential recruiter must continually readjust hiring procedures to manage a multitude of specific situations that differ from the standard situation by some contextual elements.

This present work initiates a research for improving the process of hiring potential candidates based on a Contextual Graphs Formalism. An example of a typical procedure for visualization of recruiting steps will be contextualized to add contextual elements and practices in order to communicate the recruitment policy in a concrete and memorable way to both hiring teams and candidates. An evaluation of the effect of HR practices on organizational commitment can be found in [22]. The main advantage of Contextual Graphs is the possibility to enrich incrementally the system with new knowledge and practice learning capability when needed. The paper begins by introducing an example of the hiring process. Then we present some software tools found in the literature and online. After this we show how to contextualize the hiring procedure and add practices using the Contextual Graphs formalism. Finally, we summarize the advantages of using Contextual Graphs in recruiting candidates before the conclusion of the paper.

\section{Description of the Hiring Process}

The hiring process starts when a company identifies the requirements to fill a position and ends when a candidate accepts a job offer [5]. A hiring process procedure is a visualization of recruiting steps and how they connect. It presents the recruiting steps to hiring managers in order to communicate the recruitment policy in a concrete and memorable way to both hiring teams and candidates. Figure 1 shows a basic example of a snapshot of the procedure of hiring process. 
International Journal of Artificial Intelligence and Applications (IJAIA), Vol.11, No.5/6, November 2020

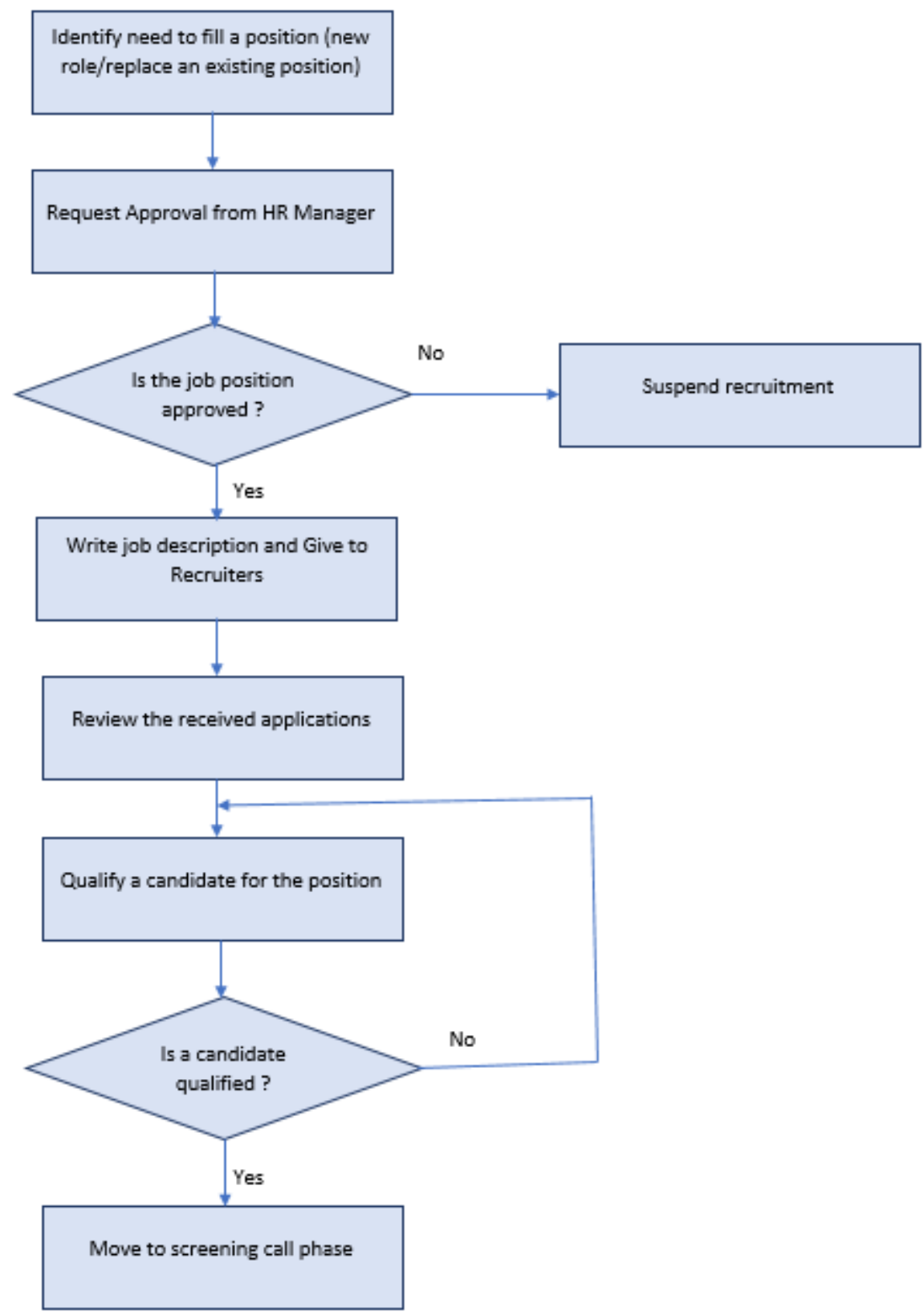

Figure 1. An example of a Hiring Process Procedure.

The main steps of the recruitment and selection process differ depending on the role and company. The main phases of a hiring process are:

Phase of planning: Hiring resource managers usually must get the job opening approved before posting the job ad. They should also consider the recruiting budget for their position, describe the job and assemble their hiring team.

Phase of attracting candidates: This phase involves any action hiring teams take to fill their hiring pipeline with qualified candidates such as candidate sourcing, job advertising and asking for referrals. 
Phase of candidate screening: The interview is the most important part of selecting candidates. Hiring teams can ensure interview of the best candidates by screening calls, job application reviews and pre-employment tests help.

Phase of offering the job: If all goes well, in this phase the company sends a job offer email to the desired candidate, who accepts it. Sometimes though, candidates may reject or choose to negotiate the offer.

This hiring process procedure cannot always be efficiently performed when new context occurs (context not completely shared between the hiring members of the team, accepted candidate reject the offer). In section 4, the Contextual graphs formalism will be used to drop the limits of this procedure and add the required contextual elements to improve the hiring process. The next section lists some tools and research papers in the area of recruiting candidates.

\section{Hiring Software ToOlS}

Many recruiting Software tools are listed by S. Mondal in [20]. The author classifies theses tools as follows: Job Aggregators, Testing \& Assessment Tools, AI \& Automation Tools [26], Applicant Tracking Systems, Recruitment CRMs, Video Interviewing Tools. Our focus is about Artificial Intelligence (AI) \& Automation Tools such as Ideal, Zoom.ai.

Ideal is used to screen and shortlist candidates by analysing rich candidate information such as resumes, assessments, conversations and performance data. Textio is used to identify biased language and then suggesting alternatives to use instead (i.e. a badly written job posting)

Zoom.ai uses automated assistant software to improve and simplify the employee experience. The main feature about this tool is about scheduling meetings, transcribing calls, and creating departmental knowledge bases.

V. Senthil Kumaran and A. Sankar [23] presented EXPERT, an intelligent tool for screening candidates for recruitment using ontology mapping. This tool collects candidates, resumes and then constructs an ontology document for the features of the candidates, in order to represent job requirements as ontology to map the job requirement ontology onto the candidate ontology document in order to retrieve the eligible candidates.

There is other recent research work that gives other alternatives to the previous tools to overcome and break some limits of automation tools. One of them was presented in [10]. Cardoso et al. proposed a methodology that aims to help answer questions that may be asked about user's preferences and behaviour, extracting information that leads to improvements in existing functionality and the creation of new ones. Other research studies discussed how E-Recruiting [15] [16] and social networks can be used in improving the process of recruiting: Using Facebook as in [9], [11], [13] and [27], LinkedIn as in [3][4][12][14][17] and [28]. An evaluation of social networks for recruiting can be found in [1].

The main problem with the automated tools is that they cannot deal efficiently or never use a new context to adapt to the new situations not predicted by the software engines of these tools. For this reason, we present the Contextual graphs formalism to show how easy to handle complex hiring situations to deal with different contexts as those discussed in the introduction of this paper. The objective is to assist the resource managers to consider the continuous changes during the different phases of the hiring process. The following section describes how to contextualize the hiring process. 


\section{Contextualization of the Hiring Process}

Context is used to describe knowledge shared on physical, social, historical and other circumstances where actions or events happen. All this knowledge does not appear in the actions to execute or the events that occur but constrains action execution and event interpretation. Brézillon and Pomerol [8] consider that context is "what constrains something without intervening in it explicitly." An important consequence is that we must speak of context in relationships with a focus and thus distinguish three types of context namely, external knowledge, contextual knowledge, and proceduralized context. The external knowledge is the knowledge that has nothing to do with the current focus. The contextual knowledge is the knowledge that is more or less related to the current focus. The actor proceduralizes a part of the contextual knowledge for addressing the current focus (the proceduralized context).

It is important to distinguish data, information and knowledge that we put under the term of contextual element (CE). Data is the basic, atomic part of the context that can be acquired directly through virtual or physical sensors, such as location coordinates, people identification or weather temperature. Information is the $\mathrm{CE}$ that can be derived from data association. While the information is something that once inferred can be easily instantiated and shared between human and software agents, the contextual knowledge is personal and it is inside people's head as mental schemas that help them to interpret external events.

In the area of incident management for subway lines, Pomerol and Brézillon [21] identified two parts in a context-based reasoning, namely diagnosis and action. The diagnosis part analyzes the situation at hand and its context in order to extract the essential facts for the actions. The actions are undertaken in a predictable order to realize the desired task. Sometimes, actions are undertaken even if the situation is not completely analyzed (or even not analyzed at all). For example, a driver puts a vehicle into gear before any action or situation analysis. Diagnosis and actions constitute a continuous twofold process, not two successive phases in context-based reasoning. Moreover, actions introduce changes in the situation or in knowledge about the situation, and imply a revision of the diagnosis, and thus of the decision-making process itself. Therefore, context must be considered explicitly with knowledge and reasoning. This is the role of the Contextual-Graphs formalism.

The development of our conceptual framework leads to the implementation in Contextual Graphs of our problem of actors' cooperation in a problem solving because Contextual Graphs are a usercentered formalism [7]. A contextual graph represents the different ways to solve a problem, and each path corresponds to a practice, a way to fix the problem. It is a directed graph, acyclic with one input and one output and a general structure of spindle [6]. Figure 2 provides the definition of the elements in a contextual graph. A more complete presentation of this formalism and its implementation can be found in [6]. Elements of a contextual graph are: actions, contextual elements, activities and temporal branching.

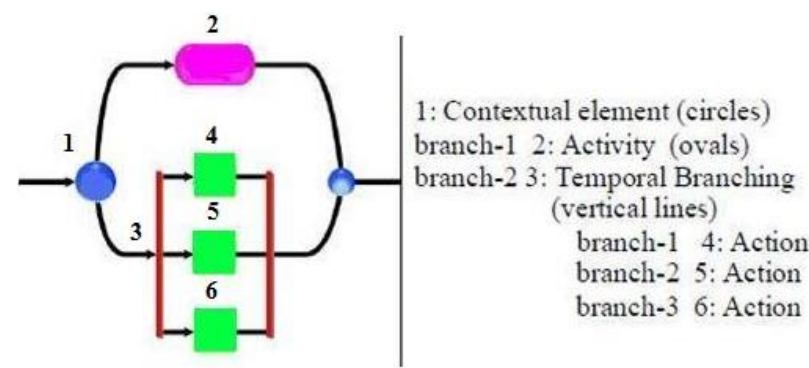

Figure 2. Elements of a contextual graph 
An action is the building block of contextual graphs at the chosen granularity. An action can appear on several paths, but it will be in different context.

A contextual element is a couple of nodes, a contextual node and a recombination node. A contextual node has one input and $\mathrm{N}$ branches $[1, \mathrm{~N}]$ corresponding to the $\mathrm{N}$ instantiations of the contextual element already encountered. The recombination node is $[\mathrm{N}, 1]$ and shows that, once the part of the practice on the branch between the contextual and recombination nodes has been executed, it does not matter to know which branch was followed.

An activity is a contextual graph by itself that is identified by participants because it appears on different paths and/or in several contextual graphs. This recurring sub-structure is generally considered as a complex action. An activity is a kind a contextualized task that can be aggregated in a unit or expanded in a sub graph according to the needs [24].

A temporal branching expresses the fact (and reduces the complexity of the representation) that several groups of actions must be accomplished but that the order in which action groups must be considered is not important, or even could be done in parallel, but all actions must be accomplished before continuing the practice development. The temporal branching is the expression of a complex contextual element at a lower granularity of the representation.

Now let us examine how to use a contextual-graph to represent the hiring process procedure discussed in section 2. Figure 3 shows the steps performed by the recruiter to find out the adequate candidate for the opened position.
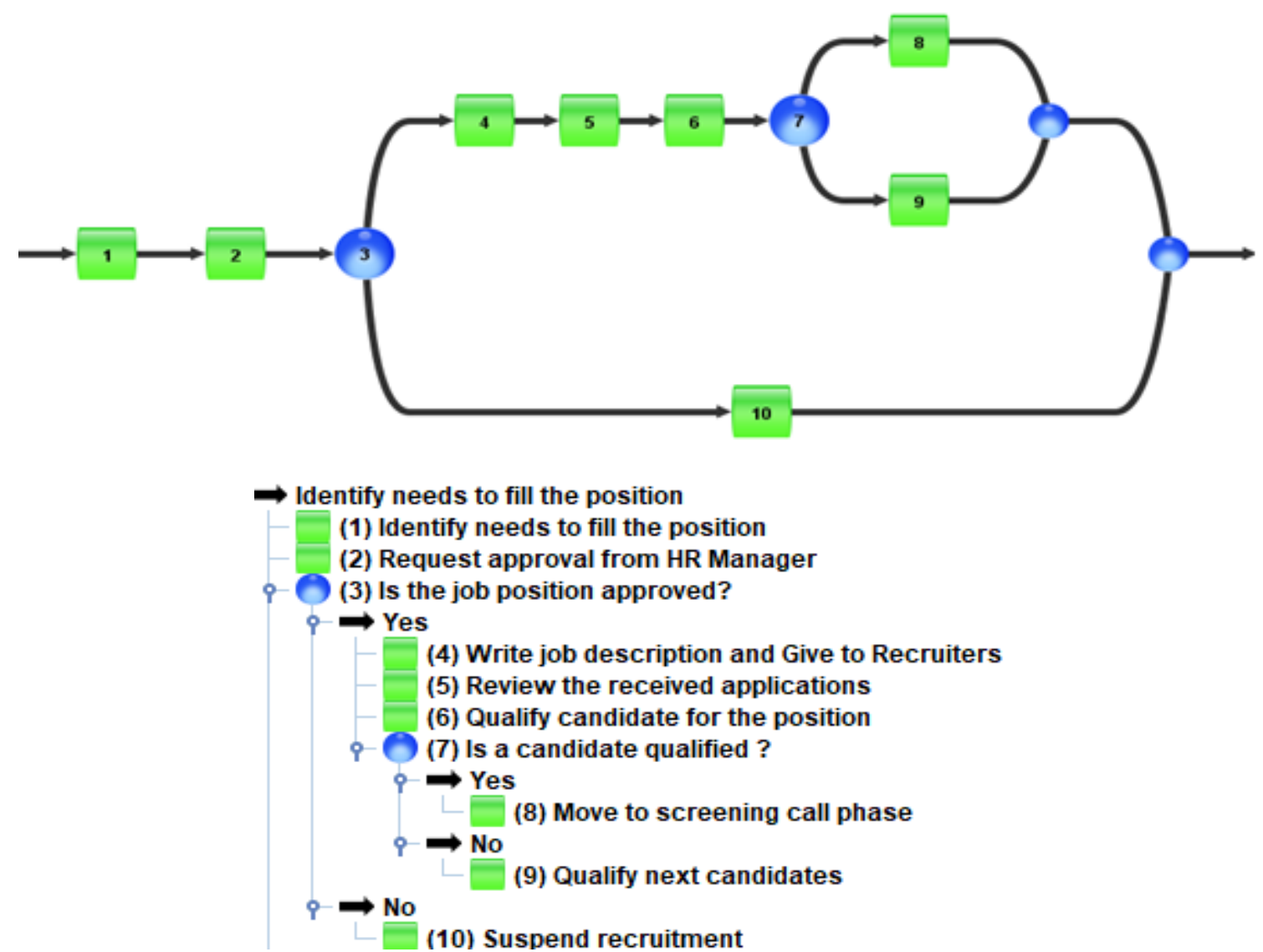

Figure 3. Contextual graph for a Hiring Process. 
This procedure should be adapted to consider the different contexts related to the given situation. A proceduralized context (PC) explains how the different items along a practice were introduced. A PC is an ordered series of instantiated contextual elements (CEs). The difference between two practices is explained through the divergence between their proceduralized contexts. Two PCs have at least a different $\mathrm{CE}$ or a same CE with different instantiations. Nodes 3 and 7 represent the contextual elements about whether or not the job is approved by the manager and if the candidate is qualified or not. In this example, actions 6,8 and 9 corresponds to the activity of executing interviews for effective qualification. These candidate interviews expose potential red flags, reveal strengths and weakness, ensure that there is a fit with salary, compensation, personality, and check technical and management qualifications, communication skills, and abilities. Activity 6 in Figure 4 represents actions 6,8 and 9 which have been executed by the interviewers according to the values of contextual elements 3 and 7.
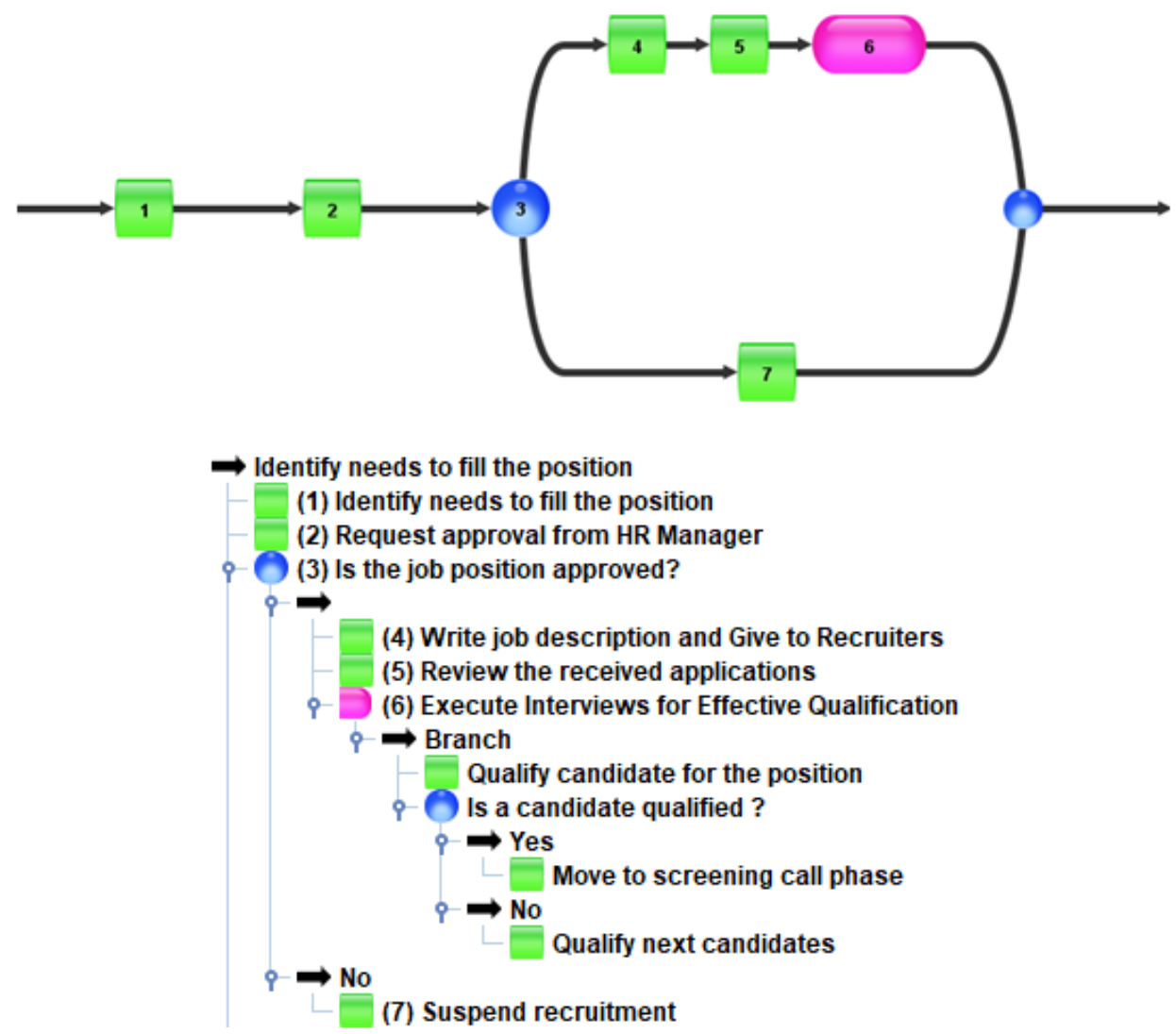

Figure 4. Contextual graph for a Hiring Process including the representation of the activity of executing interviews.

Contextual Graphs can be used to make easy screen applicants in order to select the best work position. This software tool can be used to present the criteria or the metrics that will be used when screening and identifying the potential skills of the candidates and rating each area of assessment. In addition, skill rating allows interviewers to list down all their observations in contexts related to the position and skills of the candidate. Figure 5 shows an example of contextual graphs for supporting interviewers in the hiring decision. Each path in a Contextual graph corresponds to a user practice. All the paths followed by the recruiter allow exporting the values of contextual elements and actions in order to be imported into a contextual knowledge experience database that can be used for the evaluation of candidates for best performance. 


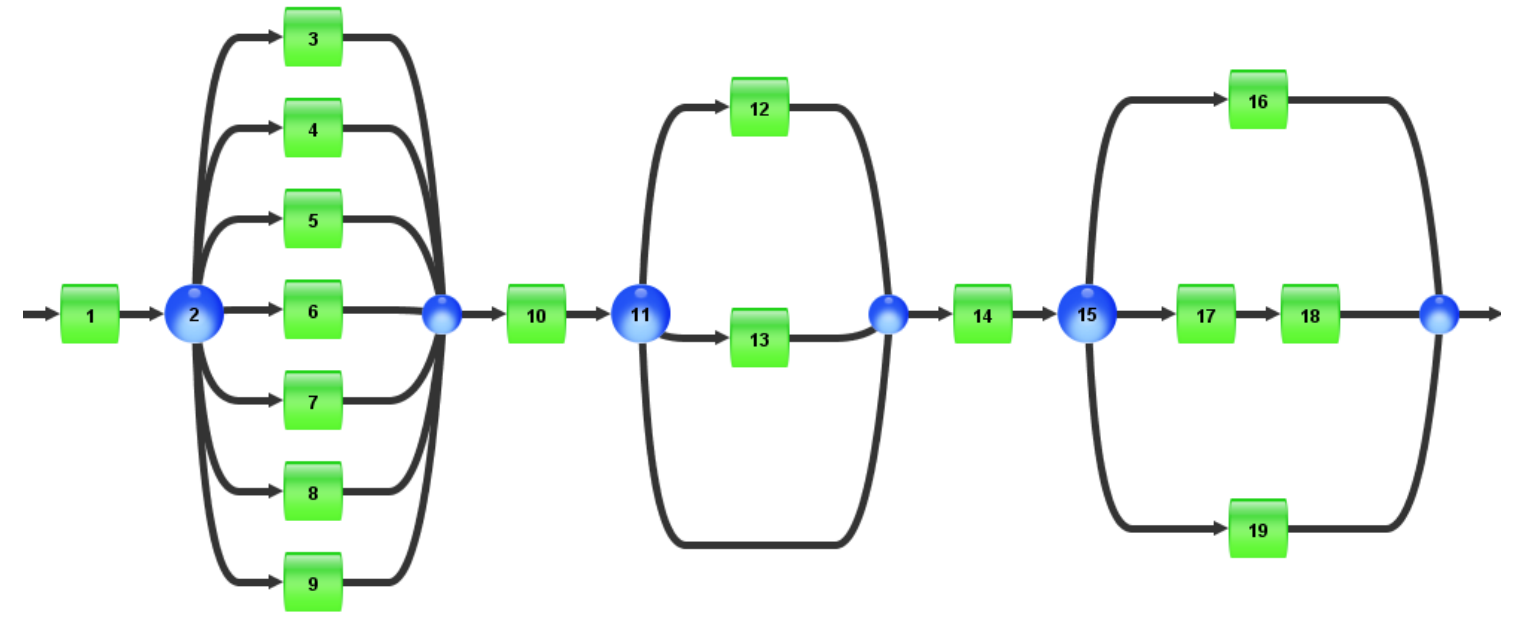

(1) Check candidate skills

p- (2) Skill Category?

$\rightarrow-$ Educational backgound

(3) Set the interview rate of the educational skills: value $=2$

$\rightarrow$ Training

(4) Set the interview rate of the training: value=2

$\rightarrow$ Prior Work Experience

(5) Set the interview rate of the prior work experience: value $=3$

$\rightarrow-$ Technical Qualifications

(6) Set the interview rate of the technical skills: value $=3$

$\rightarrow \rightarrow$ Verbal Communication

(7) Set the interview rate of the communication skills: value=1

$\rightarrow$ Management and Leadership

(8) Set the interview rate of the management skills: value $=0$

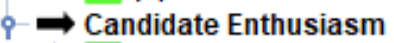

(9) Set the interview rate of the candidate skills: value=3

(10) Enter Overall rate: value $=2$

P- (11) Request an assessment test or References?

$\rightarrow$ Assessment test

(12) Evaluate candidates with an assessment test

$\rightarrow$ Reference from previous employer

$\downarrow$ (13) Check previous employers references

$\rightarrow$ No

(14) Enter a final recommendation

P (15) Type of recommendation

$\rightarrow$ Make Offer
(16) Send an offer to the candidate

$\rightarrow$ Not Chosen

(17) Indicate the reason of candidate rejection

(18) Send a candidate rejection email

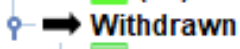

(19) Indicate the withdrawn reason

Figure 5. Contextual Graph for Supporting Interviewers in the Hiring Decision.

\section{Advantages Of USING CONTEXTUal GraPHS FOR HiRING}

Contextual Graphs can be used in the hiring process to represent the set of known practices (strategies) in order to qualify candidates. They also allow incremental acquisition of practices and provide an understandable way to model context-based reasoning. A practice is the path from the input to the output of a contextual graph. Practices take into account the various contextual 
elements about the situations faced by the recruiter. The qualification process is guided throw a specific path by the evolution of context over time.

User practices may differ from each other because of their contexts that are slightly different where users used different actions at a step of the problem solving. The process of practice acquisition by the Contextual Graphs system concerns the new action to integrate and the contextual element that discriminates that action with the previous one. The integration of the new practice requires either adding a new branch on an existing contextual node, or introducing a new contextual node to distinguish the alternatives. The phase of incremental acquisition of practices relies on interaction between the Contextual Graph system and the users in order to acquire their expertise, which consists of a context-based strategy and its evolution along the process of the hiring.

The main interest of contextual graphs relies on the possibility to introduce incrementally new practices based on cooperation between actors (Recruiters, Candidates). Now we are working to complete the architecture of the Contextual Graph Platform by designing an experience base to develop a Context-Based Intelligent System for supporting recruiters in the hiring decision.

\section{CONClusion}

This paper has initiated a research work about how a Contextual Graphs Formalism can be used for improving the decision making in the process of hiring potential candidates. We have presented an example of how to contextualize a hiring procedure and adding user practices. We have shown how making context explicit can help to improve procedures and making the best final recommendation in order to find the best possible candidate for the opened job position. Our study is in the framework of building an experience base that can be used to design an intelligent support system for recruiters. It can also be extended to several other computing areas.

\section{References}

[1] M. Amadoru, C. Gamage, Evaluating Effective Use of Social Networks for Recruitment, SIGMISCPR '16: Proceedings of the 2016 ACM SIGMIS Conference on Computers and People Research, June 2016, pp 125-133https://doi.org/10.1145/2890602.2890604

[2] Atmanco, https://atmanco.com/fr/blog/recrutement/problemes-recrutement-comment-eviter/

[3] D. Arya, V. Ha-Thuc,S. Sinha, Personalized Federated Search at LinkedIn, CIKM '15: Proceedings of the 24th ACM International on Conference on Information and Knowledge management, October 2015 Pages 1699-1702https://doi.org/10.1145/2806416.2806615

[4] M. Bastian, M. Hayes, W. Vaughan, S. Shah, P. Skomoroch, H. Kim, S. Uryasev, C. Lloyd, LinkedIn skills: large-scale topic extraction and inference,RecSys '14: Proceedings of the 8th ACM Conference on Recommender systems, October 2014, pp 1-8https://doi.org/10.1145/2645710.2645729

[5] N. BIKA, Workable Technology Limited, 2020, https://resources.workable.com/author/nikolettabika/

[6] P. Brézillon, Task-realization models in Contextual Graphs. Modeling and Using Context (CONTEXT-05), A. Dey, B.Kokinov, D.Leake, R.Turner (Eds.), Springer Verlag, LNAI 3554, pp. 55-68, 2005

[7] P. Brézillon, Representation of procedures and practices in Contextual graphs. The Knowledge Engineering Review, 18(2): 147-174, 2003.

[8] P. Brézillon and J.-C. Pomerol, Contextual knowledge and proceduralized context. Proceedings of the AAAI-99 Workshop on Modeling Context in AI Applications, Orlando, Florida, USA, July. AAAI Technical Report, 1999.

[9] R. R. Burke, B. P. Weichelt, K. Namkoong, Facebook Ads Manager as a Recruitment Tool for an Online Health and Safety Survey, Journal of Medical Internet Research, 2020

[10] A. Cardoso, F. Mourão, L. Rocha, A characterization methodology for candidates and recruiters interaction in online recruitment services, WebMedia '19: Proceedings of the 25th Brazillian 
International Journal of Artificial Intelligence and Applications (IJAIA), Vol.11, No.5/6, November 2020

Symposium on Multimedia and the WebOctober 2019, pp 333-340, https://doi.org/10.1145/3323503.3349541

[11] H. Forgasz, H.Tan, G. Leder, A. Mcleod, Enhancing survey participation: Facebook advertisements for recruitment in educational research, International Journal of Research \& Method in Education 41(3):1-14, February 2017, DOI: 10.1080/1743727X.2017.1295939

[12] H. Inan, G. Polatkan, B. Hu, Q. Guo,C. Ozcaglar,X. Wu,K. Kenthapadi, S. C. Geyik, Towards Deep and Representation Learning, for Talent Search at LinkedIn,CIKM '18: Proceedings of the 27th ACM International Conference on Information and Knowledge Management, October 2018 Pages 22532261https://doi.org/10.1145/3269206.3272030

[13] K.J. Kamp, K. Herbell, W. H. Magginis, D.L. Berry, Facebook Recruitment and the Protection of Human Subjects, Western Journal of Nursing Research 41(9):019394591982810, February w2019, DOI: $10.1177 / 0193945919828108$.

[14] K. Kenthapadi, Benjamin Le, G. Venkataraman, Personalized Job Recommendation System at LinkedIn: Practical Challenges and Lessons Learned, RecSys '17: Proceedings of the Eleventh ACM Conference on Recommender Systems, August 2017, Pages 346347https://doi.org/10.1145/3109859.3109921

[15] S. Lang, S. Laumer, C. Maier, A. Eckhardt, Drivers, challenges and consequences of E-recruiting: a literature review, SIGMIS-CPR '11: Proceedings of the 49th SIGMIS annual conference on Computer personnel research,May 2011 Pages 26-35,https://doi.org/10.1145/1982143.1982152

[16] I. Lee, An architecture for a next-generation holistic e-recruiting system, Communications of the ACM, July 2007 https://doi.org/10.1145/1272516.1272518

[17] S. Li, J. Yang, J. Yan,S. Wang, F. Chen, Q. He, Deep Job Understanding at LinkedIn, SIGIR '20: Proceedings of the 43rd International ACM SIGIR Conference on Research and Development in Information Retrieval, July 2020 Pages 2145-2148https://doi.org/10.1145/3397271.3401403

[18] A. Massaro, A. Leogrande, P. Lisco, A. Galiano and N. Savino, Innovative Bi Approaches and Methodologies Implementing A Multilevel Analytics Platform Based on Data Mining and Analytical Models: A Case of Study in Roadside Assistance Services, International Journal on Soft Computing, Artificial Intelligence and Applications (IJSCAI), Vol.8, No.1, February 2019

[19] A. Massaro, V. Vitti, P. Lisco, A. Galiano and N. Savino, A Business Intelligence Platform Implemented in a Big Data System Embedding Data Mining: A Case of Study, International Journal of Data Mining \& Knowledge Management Process (IJDKP), Vol.9, No.1, January 2019

[20] S. Mondal, The 38 Top Recruiting Software Tools Of 2020, https://ideal.com/top-recruiting-software/

[21] J.-Ch. Pomerol, P. Brézillon, Context proceduralization in decision making. In: Modeling and Using Context (CONTEXT-03), P. Blackburn, C. Ghidini, R.M. Turner and F. Giunchiglia (Eds.). LNAI 2680, Springer Verlag (http://link.springer.de/link/service/series/0558/tocs/t2680.htm). pp. 491-498, 2003.

[22] A. A. Şendoğdu, A. Kocabacak, Ş. Güven, The Relationship between Human Resource Management Practices and Organizational Commitment: A Field Study, November 2013, Procedia - Social and Behavioral Sciences 99:818-827, DOI: 10.1016/j.sbspro.2013.10.553

[23] V. Senthil Kumaran, A. Sankar, Towards an automated system for intelligent screening of candidates for recruitment using ontology mapping EXPERT, International Journal of Metadata, Semantics and OntologiesMay 2013.

[24] J.F. Sowa, Knowledge Representation: Logical, Philosophical, and Computational Foundations, Brooks Cole Publishing Co., Pacific Grove, CA, 2000.

[25] H. Tahir and P. Brézillon, Procedure contextualization for collaborative database administration. Proceedings of the 15th International Conference on Computer Supported Cooperative Work in Design, Lausanne, Switzerland, June 8-10, 2011.

[26] S. Tangadle Gopalakrishna1 and V. Varadharajan, Automated Tool for Resume Classification Using Sementic Analysis, International Journal of Artificial Intelligence and Applications (IJAIA), Vol.10, No.1, January 2019

[27] T. Welch, Is Facebook a viable recruitment tool, Nurse researcher 28(6), October 2019, DOI: 10.7748/nr. 2019.e1682

[28] X. Yan, J. Yang, M. Obukhov, L. Zhu, J. Bai, S. Wu, Q. He, Social Skill Validation at LinkedIn, KDD '19: Proceedings of the 25th ACM SIGKDD International Conference on Knowledge Discovery \& Data Mining, July 2019 Pages 2943-2951https://doi.org/10.1145/3292500.3330752 